Variation in drivers' cognitive load. Effects of driving through village areas and rural junctions

Lisbeth Harms

Reprint from Ergonomics, 1991, Vol 34, No 2, pp 151-160 
ERGONOMICS, 1991, VOL. 34, NO. 2, 151-160

\title{
Variation in drivers' cognitive load. Effects of driving through village areas and rural junctions
}

\author{
LISBETH HARMS \\ Swedish Road and Traffic Research Institute, VTI S-58101 Linköping. Sweden \\ Keywords: Driving; Dual-task performance; Cognitive load.
}

\begin{abstract}
Two experiments were performed in which drivers' cognitive load was measured by the time taken to complete calculation tasks presented to them while driving on prespecified routes. Experiment 1 was conducted on two different driving routes. Both routes included driving on a highway and driving through a village area. Mean driving speed was lower and mean calculation time was higher in the village areas than on the adjacent highway sections of the two driving routes. Across successive $100-\mathrm{m}$ intervals of the two driving routes, drivers' cognitive load was found to be inversely related to their driving speed. Experiment 2 tested the same dual-task method on driving routes including two rural junctions. At both junctions driving speed decreased with decreasing distance to the give-way line and the drivers' cognitive load was highest while approaching and driving through the junctions. It is concluded that the applied dual-task method is sensitive to the variation in drivers' cognitive load caused by variations in the demands of the driving task.
\end{abstract}

\section{Introduction}

Does driving in different traffic environments induce measurable variations in drivers' cognitive load? Following Shiffrin and Schneider (1977) and Schneider and Shiffrin (1977), two qualitatively different types of information processing strategies can be distinguished. Controlled processing is 'a temporary sequence of nodes activated under control of, and through attention by, the subject'. Controlled processing is capacity-limited and is usually serial. It is flexible, but being capacity-limited its efficiency is highly dependent on load. In contrast automatic processing denotes 'the automatic activation of a sequence of nodes by a certain stimulus configuration'. Consistent practice is of crucial importance to the development of automatic processing. Once established, automatic processing proceeds without attentional control and without stressing the capacity limitations of the system.

Driving skill has been associated with automatic processing developed through driving practice (Summala 1988), but the flexibility in drivers' responses and the variability of traffic environments suggest that driving in real traffic may require controlled processing. Consequently, variations of traffic environments may be expected to induce measurable variations in drivers' cognitive load.

\section{Previous studies}

Measurements of variations in drivers' processing capacity by dual-task methods have been conducted by a number of authors (Brown 1965, 1967, 1968, Brown and Poulton 1961, Wiegand 1974, see also Ogden et al. 1979 and Hicks and Wierwille 1979). The present study used mental calculation as a secondary task 
for the measurement of drivers' cognitive load. The time taken by the driver to complete a calculation task was measured and related to positions on prespecified driving routes.

The study is a continuation of a previous study described by Harms (1986). It aimed at testing the generality of the previous results by repeating the measurements in driving environments similar to those studied previously and by adding measurements of cognitive load in driving environments being different from those.

The previous study (Harms 1986) included three different driving routes. Each driving route consisted of a highway section and a village section. On each driving route the mean speed was lower and the mean calculation time was greater on the sections of the driving routes lying within the borderlines of a village area than it was on the adjacent highway sections. Across successive 100 -m intervals of each route, a negative correlation was found between mean driving speed and mean calculation time. Within the borderlines of the village areas, high cognitive load in 100-m intervals was associated with a high number of reported accidents in the same intervals. No such relationship was found between driving speed and number of reported accidents.

\section{Experiment 1}

Experiment 1 was undertaken to test the generality of the effects found in the previous study (Harms 1986). It was performed two years later on two of the same driving routes that had been used for the previous study. In the time period between the two experiments, the sections of the driving routes lying within the borderlines of the village areas had been reconstructed in accordance with principles of milieu-adapted through traffic (Herrstedt 1988). The reconstruction aimed to reduce the driving speed of the through traffic in the village areas and to improve the way through traffic interacted with the internal village traffic. The legal speed limit on the highways $-80 \mathrm{~km} / \mathrm{h}$-was the same for both experiments but nationwide the legal speed limit in built-up areas had been lowered from $60 \mathrm{~km} / \mathrm{h}$ to $50 \mathrm{~km} / \mathrm{h}$ in the time period between the two experiments.

\subsection{Method}

3.1.1. Subjects: Nineteen professional drivers recruited from a private salvage organization participated in the driving experiment. Nine served in Area 1 and ten in Area 2.

3.1.2. Driving task: Both driving routes included highway driving and driving through a village area. The routes had fixed locations for starting and stopping on the highway. Route lengths were $5.8 \mathrm{~km}$ in Area 1 which included $1.7 \mathrm{~km}$ in the village area, and $3.7 \mathrm{~km}$ in Area 2 which included $1.2 \mathrm{~km}$ in the village area. The drivers were instructed to drive as they 'usually would do' and to perform the calculation tasks at their own pace. All subjects performed one test trial before the first experimental session.

3.1.3. Calculation task: The drivers' cognitive load was measured by the time taken to complete a calculation task. The task was to subtract a smaller number from a greater number in a spoken two-digit number. A random sequence of the numbers from 20 to 99 was stored on a tape. The two-digit numbers were presented to the subjects one by one over headphones. An electronic control 
device stopped the tape after playing a stimulus number and restarted the tape after the termination of the subject's verbal answer. The presentation of each stimulus number followed the termination of the answer to the previous one with a delay of approximately $500 \mathrm{~ms}$.

3.1.4. Apparatus: The experimental car (Open Kadett 1.3, 1982) was equipped with two tape recorders: one (Sony TCM-134) played the random two-digit number tape over the driver's headphones (Sennheiser HME 410c, with microphone); the other tape recorder (Sony TC-D5M) was used solely for recordings. The first tape recorder started and stopped automatically when playing the numbers, and the other recorded continuously throughout the driving session. Drivers' verbal responses were recorded on one track, questions (two-digit numbers) and signals for successive $2 \cdot 6-\mathrm{m}$ intervals of driving were recorded on the other track.

Coding included time registration of recorded position signals, stimuli and responses. Driving speed was calculated for each $2 \cdot 6-\mathrm{m}$ interval, and calculation times (stimulus-off/response-on intervals) were computed and located to the position nearest the stimulus-off time. Analysis of driving speed and calculation time was based on 70 runs with a total of 10700 registered calculation times in Area 1 and 93 runs with a total of 13880 calculation times in Area 2.

\subsection{Results}

As shown in figure 1, mean driving speed was lower in the village area than on the highway on both driving routes, but mean calculation time was higher in the villages than on the highways. The speed reduction between highway and village areas was seen for all subjects. By sign test the effect was found to be significant $(p<0.001)$. The increase in mean calculation time found for 17 of the 19 subjects was also found to be significant $(p<0.01)$.

As can also be seen in figure 1, the results obtained in Experiment 1 were consistent with those of the previous experiment (Harms 1986), but two differences may be noted. First, within the village areas, driving speed was lower in the present study than in the previous one. In Area 1, mean driving speed in the village area decreased from 16.3 to $14.2 \mathrm{~m} / \mathrm{s}$ ( 59 to $51 \mathrm{~km} / \mathrm{h}$ ) between the two studies; in Area 2, mean driving speed in the village area decreased from 14.6 to $12.3 \mathrm{~m} / \mathrm{s}$ ( 53 to $44 \mathrm{~km} / \mathrm{h}$ ). Both the mean driving speed and the speed difference between the previous study and Experiment 1 were fully consistent with the observed mean speeds of passenger cars in the same village areas and time periods in studies using automatic speed registration (see Borges et al. 1987 and Borges et al. 1988). Second, mean calculation times were lower in the present study than in the previous one. This effect was found both on the highways and in the village areas. It was probably due to an undesired miscalibration of the time registration apparatus between the two studies.

Variance of calculation times varied considerably between subjects. To take account of this variation, detailed analyses of calculation times were undertaken after normalizing reaction times for individual subjects (i.e., converting them to z-scores for each subject individually) before averaging over subjects.

Figure 2 shows the mean driving speed $(\mathrm{m} / \mathrm{s})$ and the mean z-score for 34 successive $100-\mathrm{m}$ intervals of the driving route in Area 1. Figure 2 shows that driving speed was high on the highway, lower near the borderline between the 

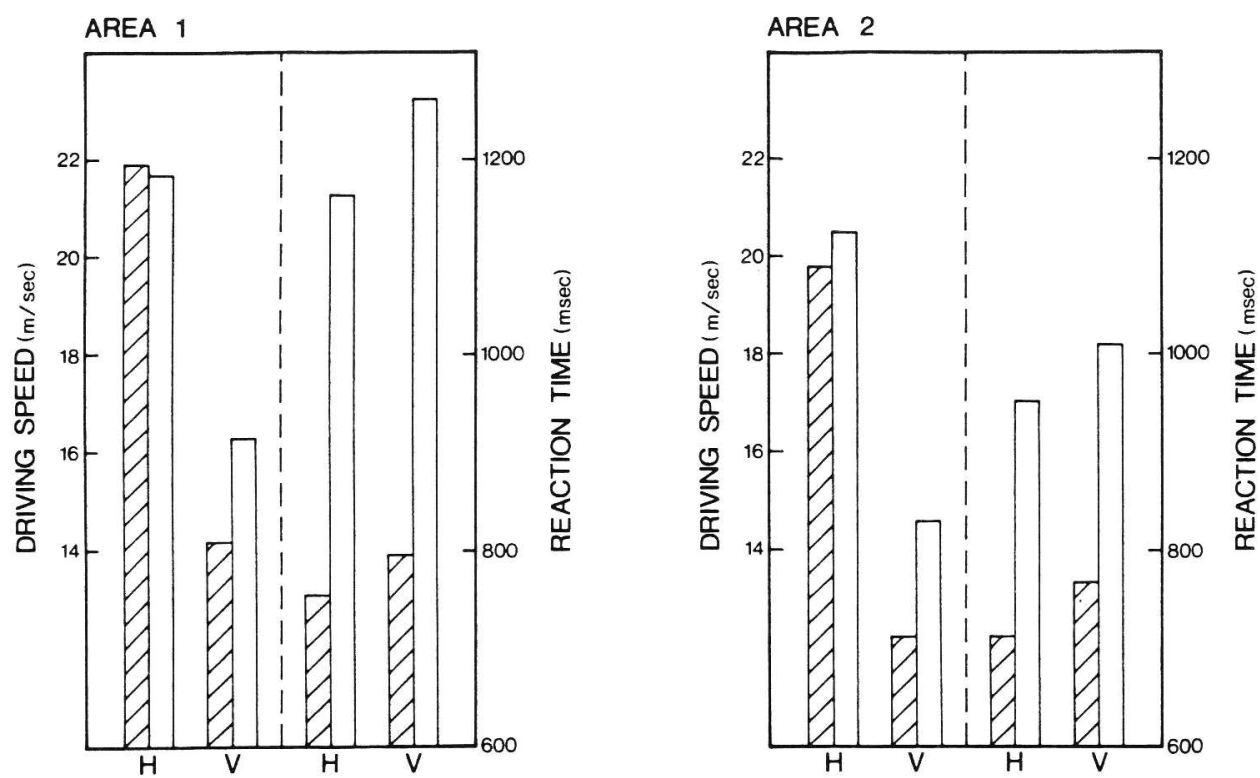

Figure 1. Mean driving speed and mean reaction time to the calculation task on the highways $(\mathrm{H})$ and in the village areas $(\mathrm{V})$ of Area 1 and Area 2.

Results of the previous study (Harms 1986) are indicated by open columns, hatched columns indicate the results of Experiment 1.

highway and the village, and lowest in the central part of the village. Calculation times were low on the highway section, higher near the borderline between the highway section and the village, and highest in the central part of the village. Roughly speaking the pattern of z-scores was a mirror image of the pattern of driving speeds. The product moment correlation between mean driving speed and calculation time (mean z-score) was -0.56 (compared to -0.61 for the same driving route in the previous study).

Figure 3 shows the mean driving speed and the mean z-score for successive intervals of Area 2. As can be seen the general pattern of results was similar to the pattern for Area 1. The product-moment correlation between driving speed and calculation time in Area 2 was -0.60 (compared to -0.76 for the same driving route in the previous study).

\subsection{Discussion}

Taken as a whole, the results obtained in both the previous study and in Experiment 1 are consistent with the assumption that variation in traffic environments induce measurable variations in drivers' cognitive load. Drivers' cognitive load was higher in the village areas than on the highway sections of the driving routes whereas driving speed was considerably lower in the village areas than on the highways. The difference in the legal speed limit between these sections of the driving routes may have determined the speed level in a general way, but the variations in driving speed between the $100-\mathrm{m}$ intervals of the driving routes indicated that environmental variation also influenced the driving speed. 


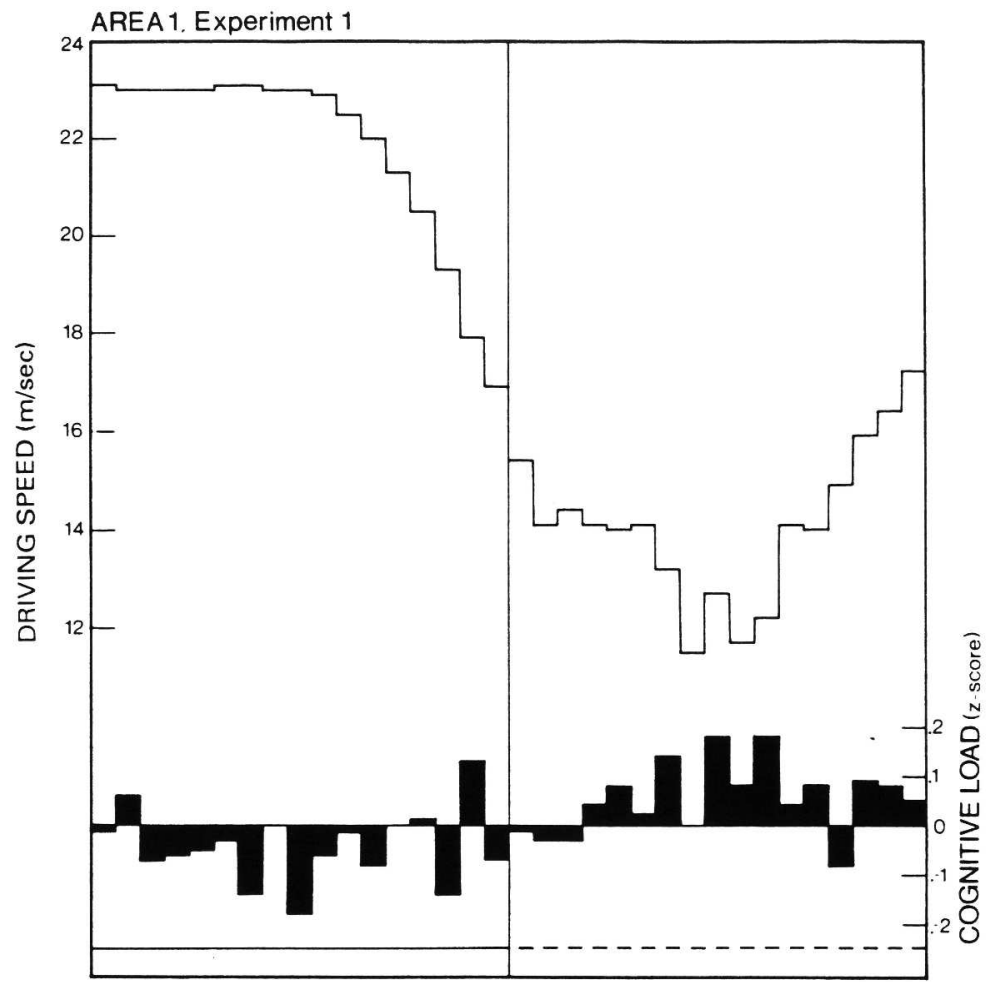

Figure 2. Mean driving speed and mean cognitive load (z-scores) for each 100-m interval of the driving route in Area 1.

The solid line denotes the highway section of the driving route; the dashed line denotes the driving route through the village.

Calculation times increased on the sections of the driving routes within the borderlines of a village area. This result was obtained in both the previous study, undertaken before the reconstruction of the driving routes, and in Experiment 1. The findings suggest that the complexity of the driving environment in the village areas, i.e., the density traffic signs, the variety of traffic flow patterns and the number and types of road users, induced a high load on the drivers' limitedcapacity processing system although the driving speed was low in these areas. The driving environments on the highways allowed both a higher speed and a higher calculation rate than did those in the village areas.

The consistent results obtained on the three different driving routes in the previous study and on both of the driving routes in Experiment 1 strongly suggest that the dual-task method used was sensitive in measuring variations in drivers' cognitive load in response to the demands of the driving task.

\section{Experiment 2}

Experiment 2 tested the assumption that variations in cognitive load are general effects of variation in the demands of the driving task that can also be found in other traffic environments. It was predicted that variations in driving speed and cognitive load similar to the variations found when driving through 


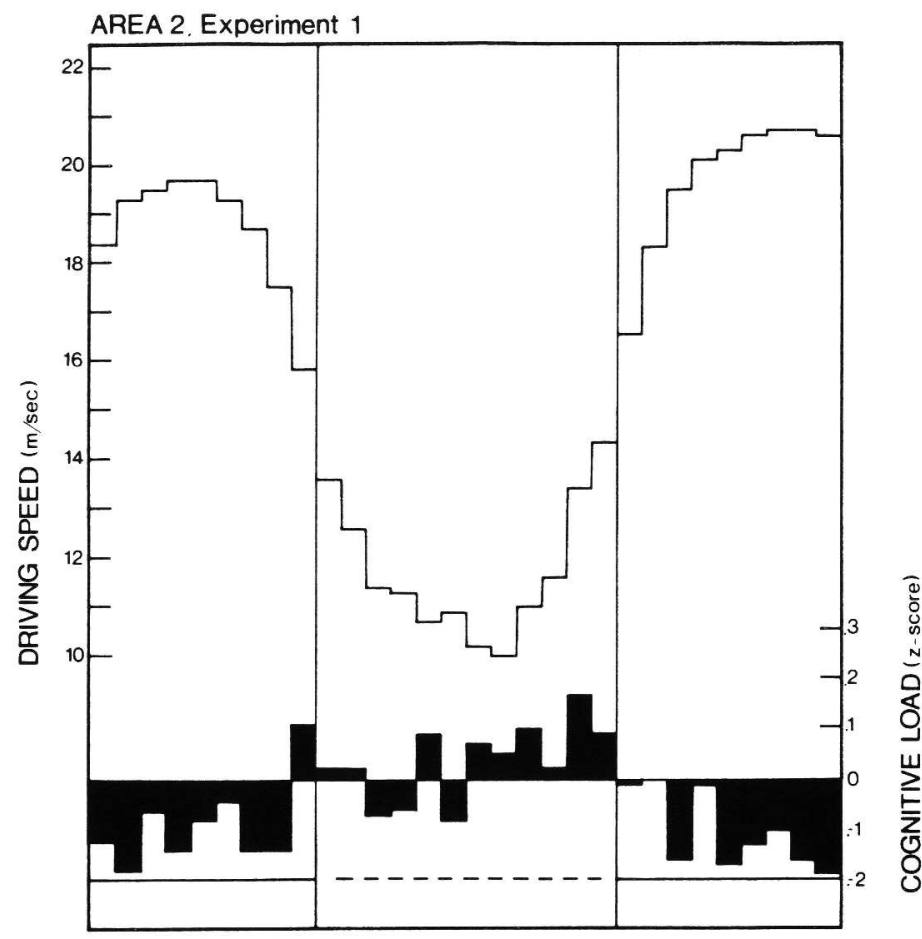

Figure 3. Mean driving speed and mean cognitive load (z-scores) for each 100-m interval of the driving route in Area 2.

The solid line denotes the highway sections of the driving route; the dashed line denotes the driving route through the village.

a village area, would also be found on driving routes leading through rural junctions.

Free sight before the give-way line at rural junctions may provide the driver with sufficient information to continue driving without pausing at the give-way line, unless the passage of other vehicles must be awaited. The give-way line is normally passed at a low driving speed whether the driver passes unhindered through a junction or pauses. However, it may be questioned whether a driver's speed reduction or even a stop at the give-way line can prevent an increase in cognitive load due to the increase in the task demands associated with driving through a junction.

\subsection{Method}

The method was essentially the same as that used in Experiment 1.

4.1.1. Subjects: Fourteen non-professional drivers from a local administration office volunteered for the driving experiment.

4.1.2. Driving task: The driving routes selected for the experiment were minor roads passing through two 4-way rural junctions. They were approximately $2 \mathrm{~km}$ apart on the same highway. At both junctions the view was clear before passing the give-way line. Both the junctions were passed in every possible fashion, i.e., 
left-turn manoeuvres, right-turn manoeuvres or driving straight through the junctions.

Analyses of mean driving speed and mean reaction times to the calculation task included a total of 98 passages through Junction 1 with a total of 1010 reaction times and 133 passages through Junction 2 with a total of 1321 reaction times. Of the total of 231 passages, 64 were left-turn manoeuvres, 76 were rightturn manoeuvres, and 91 were runs straight through a junction.

\subsection{Results}

The analyses of mean driving speed and mean calculation time (z-scores) at the two junctions were limited to three 50-m intervals of the driving route before the give-way line, the $50-\mathrm{m}$ interval including the give-way line and three $50-\mathrm{m}$ intervals after the give-way line. Mean driving speeds and mean calculation time (z-scores) were computed for each subject individually before averaging over subjects.

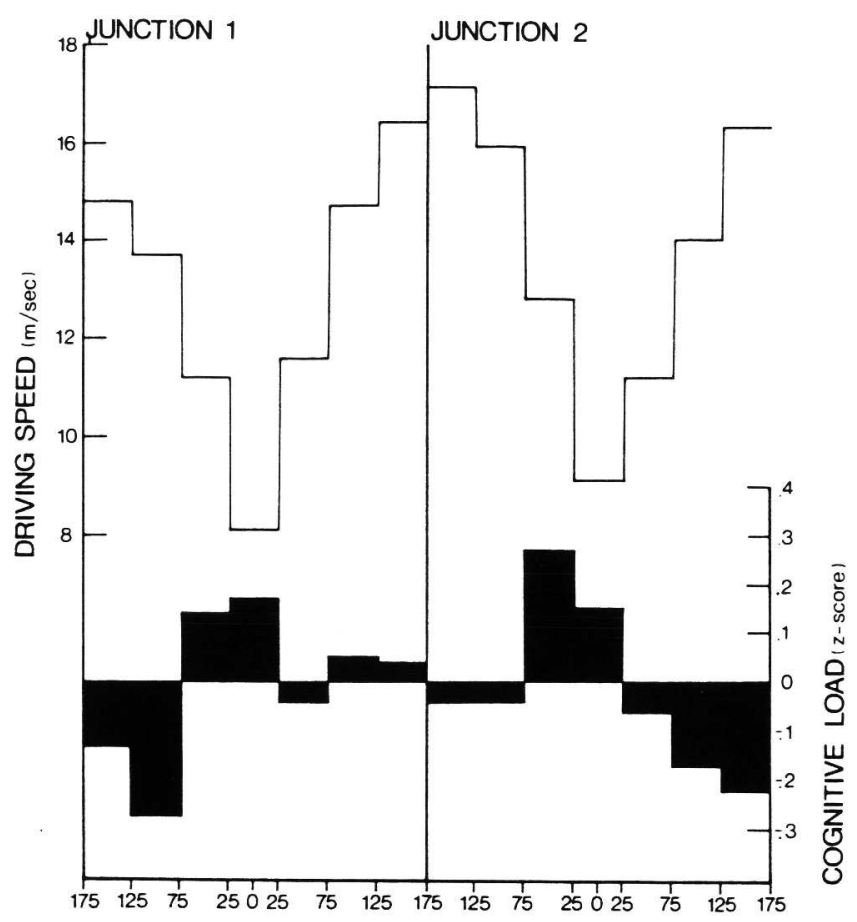

Figure 4. Mean driving speed and mean cognitive load (z-scores) in junction 1 and junction 2.

The distance to the give-way line is indicated in metres. The give-way line is indicated by zero.

As can be seen from figure 4 mean driving speed decreased with decreasing distance to the give-way line in both junctions. The lowest driving speed was measured in the 50-m interval including the give-way line. Mean calculation time was inversely related to driving speed, as was the case in the previous study and in Experiment 1. At both junctions the highest mean calculation time was 
found in the 50-m interval including the give-way line and in the preceding $50-\mathrm{m}$ interval, where the drivers had a clear view. This effect-greater calculation time in these two interval compared to other interval observed-was found for 11 of the 14 subjects $(p<0.05$ by sign test).

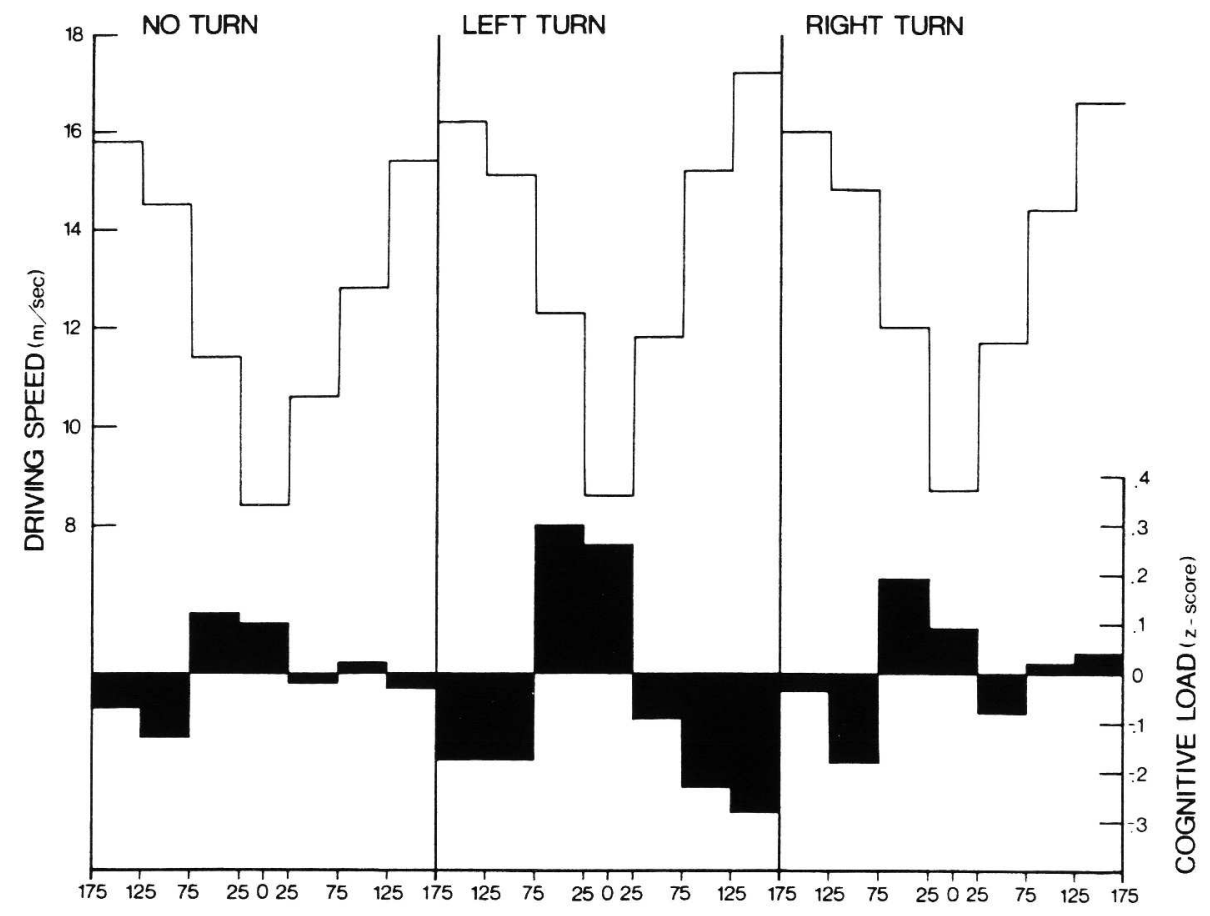

Figure 5. Mean driving speed and mean cognitive load (z-scores) for the three different driving manoeuvres (no-turn, left-turn and right-turn).

The distance to the give-way line is indicated in metres. The give-way line is indicated by zero.

As shown in figure 5 the mean z-score for the two critical intervals (the interval including the give-way line and the immediately preceding interval) was considerably higher for left-turn manoeuvres than for either right-turn or no-turn manoeuvres.

With right-hand traffic, left-turn manoeuvres are usually considered more demanding at junctions than are both right-turn and no-turn manoeuvres, and mean cognitive load was highest for left-turn manoeuvres. However, as might have been expected from the fact that traffic situations varied between drives through the junctions, this effect was not consistent across drives. It was based on a few runs with very long calculation times associated with left-turn manoeuvres.

\section{General discussion}

The results obtained in both the previous study (Harms 1986) and in the current two experiments showed that variations in drivers' cognitive load in response to variation of traffic environments can be measured by the present dual-task 
method. In both the previous study and in the current experiments the variations in drivers' cognitive load were related to the demands of the driving task caused by variations in the driving environment of the observed driving routes.

In all the experiments an inverse relationship was found between driving speed and cognitive load, as measured by the drivers' calculation times. Presumably, low task demands on highways allowed the drivers to both perform the calculation task at a high rate and to drive at a high speed. Processing demands arising from the traffic environments of village areas and rural junctions were higher and caused a substantial increase in the drivers' cognitive load although the drivers actually reduced their speed in those environments.

The difference between the legal speed limit in the villages and on the highways may have contributed to the pattern of results obtained. Without legal speed limits the drivers might have maintained a higher driving speed on the highways and this might have increased their cognitive load on the highway sections of the driving routes. Presuming that the speed in the complex environments of village areas and at the junctions would have been at the same level, it may be assumed that the inverse relationship between driving speed and cognitive load would have been modified or even that it might have disappeared.

On the other hand, it is questionable whether variations in drivers' cognitive load in real traffic can be compensated thoroughly by speed reductions. Traffic environments may cause a high cognitive load even if the driving speed is extremely low.

The relationship between cognitive load and number of registered accidents was not investigated in the current study. Findings from the previous study (Harms 1986) suggested that variations in drivers' cognitive load were related to driving safety.

Both the previous study and the current study referred to variations in the driving environment that indicated a difference in the driving task. The method was sensitive to the influence of these differences on drivers' cognitive load. The dual-task method may even prove to be useful for general assessments of the influence of driving environments on drivers' cognitive load.

\section{Acknowledgements}

The experimental studies were conducted at the Danish Council of Traffic Safety Research. The publication of the results was supported with grants from the Nordic Committee on Road Safety Research (NKT). Thanks also to anonymous reviewers for helpful criticism and advice.

\section{References}

Borges, P., Andersen, P. S., Herrstedt, L. and Fjeldsted, P. 1987, Consequence evaluation of enviromentally adapted through road in Vinderup, Report 52, Road Data Laboratory, Danish Road Directorate.

Borges, P., Andersen, P. S., Herrstedt, L. and Fjeldsted, P. 1988, Consequence evaluation of environmentally adapted through road in Skærbæk, Report 63, Road Data Laboratory, Danish Road Directorate.

BRown, I. D. 1965, A comparison of two subsidiary tasks used to measure fatigue in car drivers, Ergonomics, 8, 467-473.

BRown, I. D. 1967, Measurements of control skills vigilance and performance on a subsidiary task during 12 hours of car driving, Ergonomics, 6, 229-233. 
BRown, I. D. 1968, Some alternative methods of predicting performance among professional drivers in training, Ergonomics, 1, 13-21.

BRown, I. D. and Poulton, E. C. 1961, Measuring the spare mental capacity of car drivers by a subsidiary task, Ergonomics, 1, 31-40.

HARMS, L. 1986, Drivers' attentional response to environmental variations: a dual-task real traffic study. In A. G. Gale et al. (eds) Vision in Vehicles, I (North-Holland) pp 131-138.

Herrstedt, L. 1988, Environmentally adapted through-road in Vinderup-effect evaluation, Proceedings from the 14. ARRB Conference, part 2, Traffic, Canberra, 1988, pp 85-97.

Hicks, T. G. and Wierwille, W. W. 1979, Comparison of five mental workload assessment procedures in a moving base driving simulator, Human Factors, 1, 129-143.

Ogden, G. D., Levine, J. R. and Eisner, E. J. 1979, Measurement of workload by secondary tasks, Human Factors, 5, 529-548.

SCHNEIDER, W. and Shiffrin, R. M. 1977, Controlled and automatic human information processing I. Detection, search and attention, Psychological Review, 1, 1-66.

SHIFFrIN, R. M. and SCHNEIDER, W. 1977, Controlled and automatic human information processing II. Perceptual learning, automatic attending and general theory, Psychological Review, 2, 127-190.

Summala, H. 1988, Risk control is not risk adjustment: the zero risk theory of driver behaviour and its implications, Ergonomics, 4, 491-507.

WiEgand, D. 1974, Die quantitative Messung der psychischen Beanspruchung während des Farhrens durch eine simultane Nebentätigkeit, Zeitschrift für Experimentelle und Angewandt Psychologie, 4, 679-690.

Manuscript received 24 April 1990.

Manuscript accepted 5 May 1990. 


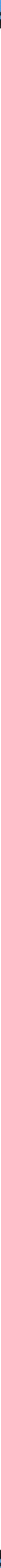

